\title{
Time-dependent search of high energy cosmic neutrinos from variable Blazars with the ANTARES telescope
}

\author{
Agustín Sánchez-Losa* \\ IFIC - Instituto de Física Corpuscular, Edificios Investigación de Paterna, CSIC - Universitat de \\ València, Apdo. de Correos 22085, 46071 Valencia, Spain \\ E-mail: agustin.sanchez@ific.uv.es
}

\section{Damien Dornic ${ }^{\dagger}$}

Aix Marseille Université, CNRS/IN2P3, CPPM UMR 7346, 13288, Marseille, France

E-mail: dornic@cppm.in2p3.fr

\begin{abstract}
ANTARES, the largest neutrino telescope operating in the Northern Hemisphere, performs multiple analyses in the search for neutrino point-source candidates. In a time-dependent search, the background is drastically reduced, and the point-source sensitivity improved, by selecting a narrow time window around the assumed neutrino production period. Blazars are particularly attractive potential neutrino point sources, since they are among the most likely sources of the observed very-high-energy cosmic rays. Neutrinos and gamma rays may be produced in hadronic interactions with the surrounding medium. Moreover, blazars generally show large time variability in their light curves at different wavelengths and on various time scales. For the time-window selection, their gamma ray emission measured by the LAT instrument on-board the Fermi satellite is derived, and the resulting light curves are characterised by a time series analysis. The studied periods are determined by applying a threshold on the fluence on the light curves. In addition, the flares reported at $\mathrm{TeV}$ energies by the IACTs HESS, MAGIC and VERITAS have been included in a second dedicated analysis. The sensitivities reached with this method improve by a factor 2-3 with respect to a standard time-integrated point source search. The results of the two searches, using data from the years 2008 up to 2012, will be presented.
\end{abstract}

The 34th International Cosmic Ray Conference,

30 July - 6 August, 2015

The Hague, The Netherlands

\footnotetext{
${ }^{*}$ On behalf of the ANTARES collaboration

† Speaker.
} 


\section{Introduction}

High-energy neutrino source detection would yield to identify the cosmic ray sources [1] and provide answer to the responsible acceleration mechanisms hosted on them. Active galactic nuclei $(\mathrm{AGN})$ are among these candidates, although it remains unclear if their gamma ray emissions are due to leptonic [2] or hadronic processes [3,4]. In the later case, the emission is attributed to $\pi^{0}$ decays; the corresponding production of charged pions implies a correlated neutrino emission.

Flat-Spectrum radio quasars (FSRQs) and BL Lacs, together classified as blazars [5], are among the most likely sources of the observed very high-energy cosmic rays [6, 7]. Multiple models predict different neutrino fluxes from AGNs with different normalisations and shapes [814]. The $E^{-2}$ spectrum is generally the most expected, yet some authors estimate more optimistic spectral indexes up to one $[15,16]$. Additionally, in most gamma ray sources is observed an energy cutoff. To cover the wide range of possibilities, four neutrino spectra are tested in this analysis: $E^{-1}, E^{-2}, E^{-2} \exp (-E / 10 \mathrm{TeV})$ and $E^{-2} \exp (-E / 1 \mathrm{TeV})$, with $E$ is the neutrino energy.

The ANTARES telescope [17] detects events through the Cherenkov light emitted by muons in the deep sea. To distinguish astrophysical neutrino events from background ones (atmospheric muons and neutrinos), energy and direction reconstruction of events have been used in several searches [18-20]. To improve this discrimination, the arrival time information can be used reducing significantly the effective background. Blazars present time variable emissions through different wavelengths at different time scales [21-23]. This variability would take place also in the corresponding neutrino emission. The use of this information in the time-dependant methods improve the detection probability with respect to time-integrated approaches.

The results of a time-dependent search for cosmic neutrino sources in the sky visible to the ANTARES telescope using data taken from 2008 to 2012 are presented. This extends a previous ANTARES analysis [24] where only the last four months of 2008 were considered. The analysis is applied to a list of promising AGN candidates detected flaring by the FERMI satellite, and to a list of flares reported by TeV-range experiments (H.E.S.S., MAGIC and VERITAS).

\section{Time-dependent search method}

An unbinned likelihood-ratio maximisation method is used to perform the analysis. Data are parametrised as a two-component mixture of signal and background. The probability density function $\mathscr{P}_{i}$ and the likelihood $\mathscr{L}$ are:

$$
\begin{gathered}
\mathscr{P}_{i}=\mathscr{N}_{\mathrm{S}} \mathscr{S}_{i}+\mathscr{N}_{\mathrm{B}} \mathscr{B}_{i} \\
\ln \mathscr{L}=\sum_{i=1}^{N} \ln \left[\mathscr{N}_{\mathrm{S}} \mathscr{S}_{i}+\mathscr{N}_{\mathrm{B}} \mathscr{B}_{i}\right]-\left[\mathscr{N}_{\mathrm{S}}+\mathscr{N}_{\mathrm{B}}\right]
\end{gathered}
$$

where $\mathscr{N}_{\mathrm{S}}$ and $\mathscr{N}_{\mathrm{B}}$ are the expected number of signal (unknown) and background (known) events, with probability distributions (PDFs) for an event $i, \mathscr{S}_{i}$ and $\mathscr{B}_{i}$, respectively. These PDFs are the product of three components that describe the event direction, energy and timing probabilities.

For an event $i$, the signal PDF is:

$$
\mathscr{S}_{i}=\mathscr{S}_{i}^{\text {space }}\left(\Psi_{i}\left(\alpha_{s}, \delta_{s}\right)\right) \cdot \mathscr{S}_{i}^{\text {energy }}\left(d E / d X_{i}\right) \cdot \mathscr{S}_{i}^{\text {time }}\left(t_{i}+l a g\right)
$$


where $\mathscr{S}_{i}^{\text {space }}$ represents the point spread function, $\mathscr{S}_{i}^{\text {energy }}$ is the energy PDF according to the studied energy spectrum and $\mathscr{S}_{i}^{\text {time }}$ is the time PDF, extracted from the gamma ray emission of the studied source. A possible lag of up to \pm 5 days is implemented in the likelihood to allow small lags in the gamma ray emission and the neutrino signal. This parameter is maximised in the likelihood together the number of signal events $\left(\mathscr{N}_{\mathrm{S}}\right)$. For the signal simulation, the correlation between $\mathscr{S}_{i}^{\text {space }}\left(\Psi_{i}\left(\alpha_{s}, \delta_{s}\right)\right)$ and $\mathscr{S}_{i}^{\text {energy }}\left(d E / d X_{i}\right)$ is taken into account.

The background PDF is:

$$
\mathscr{B}_{i}=\mathscr{B}_{i}^{\text {space }}\left(\delta_{i}\right) \cdot \mathscr{B}_{i}^{\text {energy }}\left(d E / d X_{i}\right) \cdot \mathscr{B}_{i}^{\text {time }}\left(t_{i}\right)
$$

where the directional PDF $\mathscr{B}_{i}^{\text {space }}$, the energy PDF $\mathscr{B}_{i}^{\text {energy }}$ and the time PDF $\mathscr{B}_{i}^{\text {time }}$ for the background are derived from data.

The significance of the analysis is determined through a likelihood ratio test statistic, $\lambda$, defined as:

$$
\lambda=\sum_{i=1}^{N} \ln \frac{\mathscr{P}\left(x_{i} \mid H_{\mathrm{sig}+\mathrm{bkg}}\left(\mathscr{N}_{\mathrm{S}}\right)\right)}{\mathscr{P}\left(x_{i} \mid H_{\mathrm{bkg}}\right)}
$$

where $\mathscr{N}_{\mathrm{S}}$ and $N$ are respectively the unknown number of signal events and the total number of events in the considered data sample. Its evaluation is performed through pseudo-experiment simulations.

Tests on the performance of the time-dependent analysis shows on-average a factor 2-3 of improvement with respect to the time-integrated case $[18,20]$.

\section{Gamma-ray flares}

\subsection{GeV flares: Fermi LAT}

The time-dependent analysis described is applied to bright and variable Fermi blazar sources reported in the second Fermi LAT catalogue [25] and in the LBAS catalogue (LAT Bright AGN sample [26]). From there, are selected the sources visible by ANTARES $\left(\delta<35^{\circ}\right)$ with a gamma ray flux greater than $10^{-9}$ photons $\cdot \mathrm{cm}^{-2} \cdot \mathrm{s}^{-1}$ above $1 \mathrm{GeV}$, a detection significance $T S>25$ and a significant time variability. This list is completed up to a total of 154 sources by including sources reported as flaring in the Fermi Flare Advocates in 2011 and 2012 [27].

The gamma ray light curves are produced using the Fermi Public Release Pass 7 data with the source class event selection (evclass=2) and the Fermi Science Tools v9r35p1 package [28], processing the photon counting data above $100 \mathrm{MeV}$, from August 2008 to December 2012, in a $2^{\circ}$ cone radius around the studied source direction. Sources close to the galactic plane (galactic latitude $\mid \mathrm{ll}<10^{\circ}$ ) or with other sources within a $2^{\circ}$ cone (or $3^{\circ}$ for very bright sources) are excluded due to different origin gamma ray contamination.

A maximum likelihood block (MLB) algorithm [29-31] is used to remove noise from the light curves by iterating over the data points and selecting periods during which data are consistent with a constant flux within statistical errors. The flaring periods are defined through a threshold on the fluence on these denoised light curves, based on the gamma ray emission baseline and flare significance. The final list which includes any gamma ray flaring source reduces to 41 blazars: 33 Flat Spectrum Radio Quasars, 7 BL-Lacs and 1 unknown identification. 


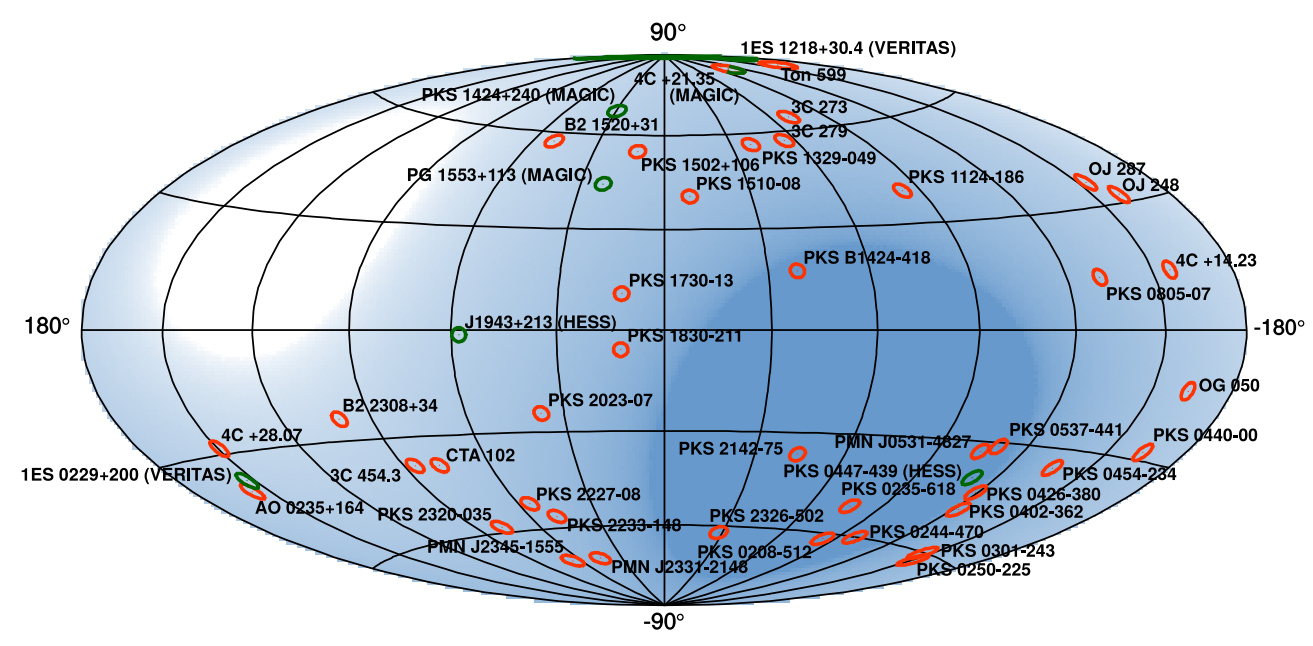

Figure 1: Skymap in galactic coordinates showing the position of the 41 selected Fermi blazars (red circle) and the $7 \mathrm{TeV}$ blazars (green circle) on top of the ANTARES visibility of the analyses $(\cos (\theta)>-0.15)$.

\subsection{TeV flares: IACTs}

Imaging air Cherenkov telescopes (IACTs) such as H.E.S.S., MAGIC and VERITAS cannot monitor sources continuously. These telescopes detect photons with energies in the GeV- $\mathrm{TeV}$ range that can be better correlated with high energy neutrinos. These observatories often emit alerts reporting flares to Astronomer's Telegram or directly in a dedicated paper. From them, the flaring periods are extracted, assuming a single square-shaped flare. The sources are chosen for this analysis according to the same visibility criteria as for Fermi/LAT observations, comprising 7 blazars. The same analysis as described previously is performed assuming the same four energy spectra.

Figure 1 shows the position of the Blazars analysed, on top of the ANTARES visibility.

\section{Results \& discussion}

Of the GeV flares, only three sources, 3C279, PKS10235-618 and PKS1124-186, have a pretrial p-value lower than $10 \%$. The lowest p-value, $3.3 \%$, is obtained for the source $3 \mathrm{C} 279$ where one event is coincident with a large gamma ray flare detected by Fermi/LAT in November 2008. This event has already been reported in a previous analysis [24]. The post-trial probability, computed by taking into account the 41 searches, is $67 \%$, and is thus compatible with background fluctuations.

Among the seven tested flares reported by IACTs, only the blazar PKS0447-439 shows a pretrial p-value lower than $10 \%$ in the case of the assumed $E^{-2} \exp (-E / 1 \mathrm{TeV})$ energy spectrum. The corresponding post trial p-value is $55 \%$, being also consistent with background fluctuations.

In the absence of a discovery, upper limits on the neutrino fluence $\mathscr{F}_{v}$ at $90 \%$ confidence level are computed using $5-95 \%$ of the energy range as:

$$
\mathscr{F}_{v}=\int_{t_{\min }}^{t_{\max }} \mathrm{d} t \int_{E_{\min }}^{E_{\max }} \mathrm{d} E \times E \frac{\mathrm{d} N}{\mathrm{~d} E}=\Delta t \int_{E_{\min }}^{E_{\max }} \mathrm{d} E \times E \frac{\mathrm{d} N}{\mathrm{~d} E}
$$




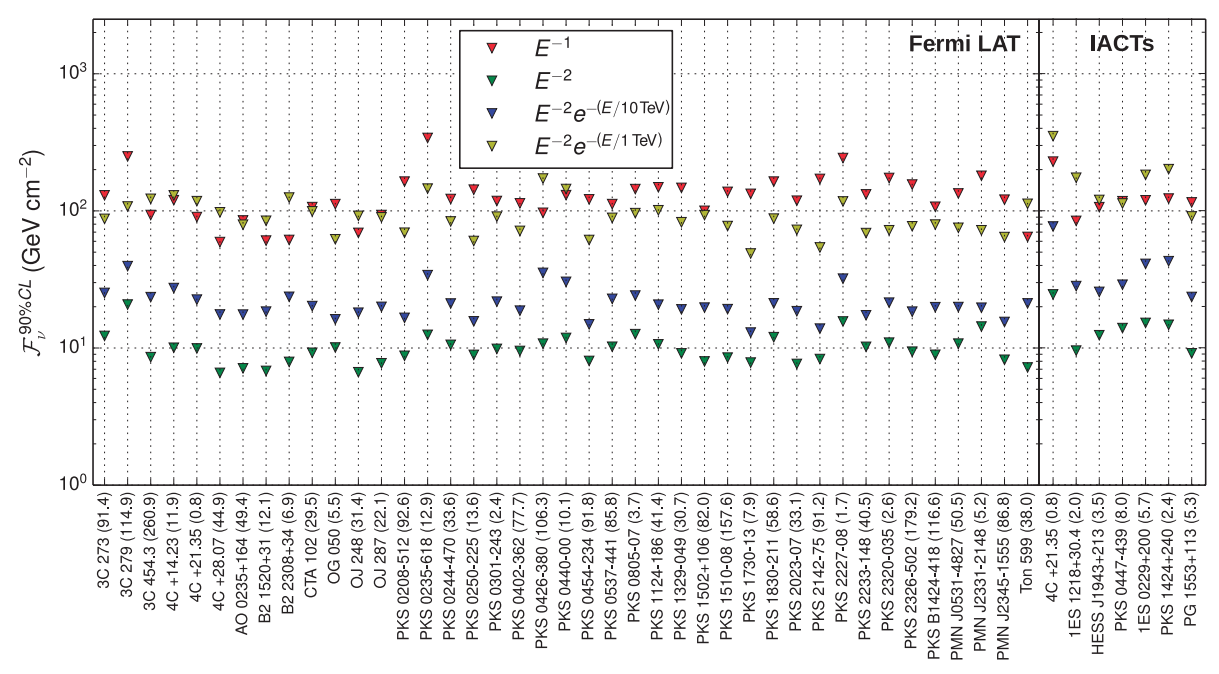

Figure 2: Upper-limits on the neutrino fluence for the 41 studied Fermi blazars (left) and the 7 studied TeV blazars (right) in the case of $E^{-2}$ (green), $E^{-2} \exp (-E / 10 \mathrm{TeV})$ (blue), $E^{-2} \exp (-E / 1 \mathrm{TeV})$ (yellow) and $E^{-1}$ (red) neutrino energy spectra. The number in paranthesis after the name of the source in the x-axis indicates the total flare length during the studied period.

The emission duration, $\Delta t$ is computed using the effective livetime during the flare. The limits include systematic errors and are calculated according to the classical (frequentist) method for upper limits [32] (see Figure 2). IceCube has performed a similar time-dependent analysis [33] using data from 2008 to 2012 with similar results. 19 sources are in common with the Fermianalysed sources. For sources in the Southern Hemisphere, the limits computed by IceCube are on the same order of magnitudes whereas the ANTARES limits are a factor 10 worse for the sources in the Northen hemisphere.

Hadronic interactions predict neutrino emission in the TeV-PeV range associated with a flux of gamma rays. The prediction that the total neutrino energy flux $F_{V}$ is approximately equal to the total high-energy photon flux $F_{\gamma}$ is relatively robust, at least when attributing this emission to a 100\% hadronic origin [36, 37]. Using spectral energy distributions (SEDs) and VHE data from the literature is possible to see how gamma ray emissions compare with the neutrino upper limits for different spectral indexes, from -3 to -1 . These limits are extrapolated from the $E^{-2}$ spectrum case by considering the proper change in the acceptance. In Figure 3 is shown the hybrid SED for the blazar 3C279. With this simple criteria of the energy budget, the limit set by ANTARES for the blazar 3C279 is on the same order of magnitude as the gamma ray flux measured during the flares. This encourages the search for a neutrino signal during outburst periods. With more data, ANTARES will be able to significantly constrain a 100\% hadronic origin of the high-energy gamma ray emission. Fermi has reported some very intense outbursts between mid 2013 and end of 2014 for $3 C 279$ [38, 39], periods not considered in this paper. 


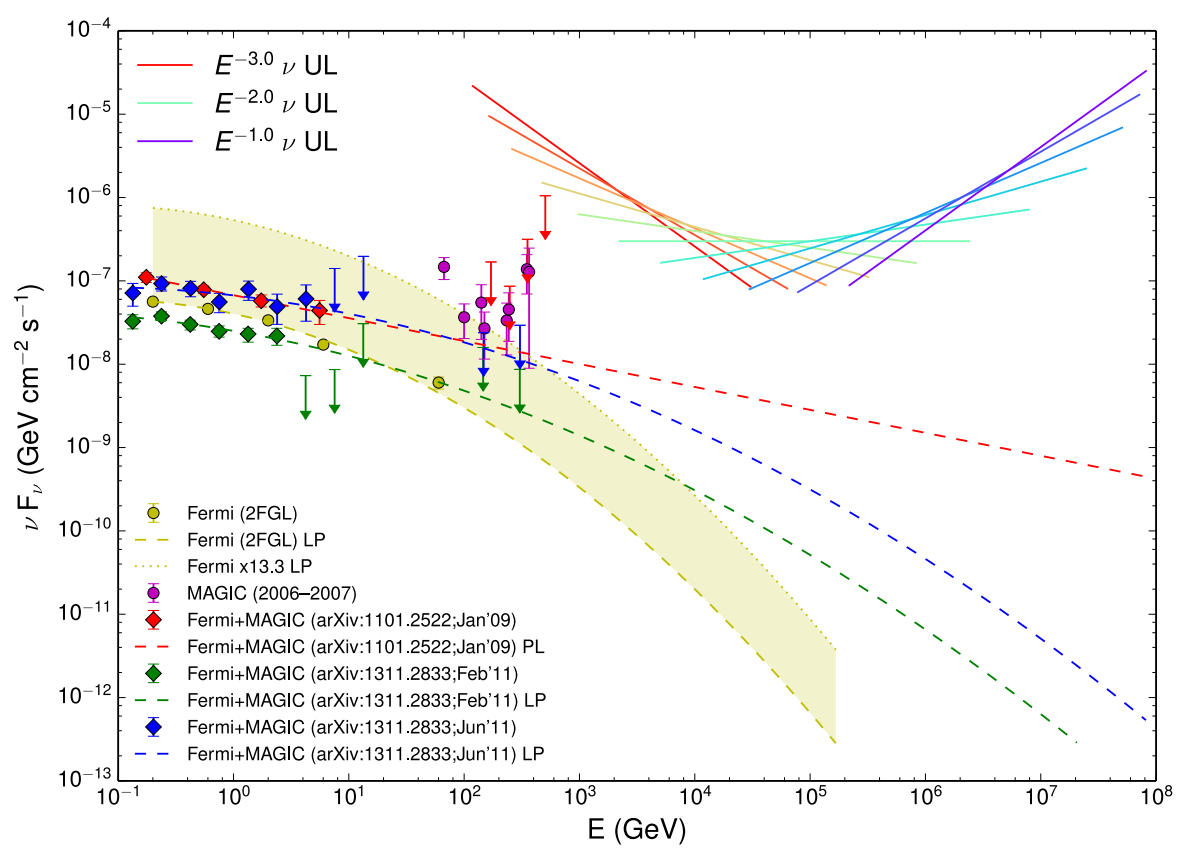

Figure 3: Gamma-ray SED of 3C279 observed by Fermi/LAT at different epoches: red, green and blue dots for measurements in January 2009, January 2011 and June 2011 respectivelly [34, 35]. The yellow dots are the average flux with 2008-2010 data (2FGL). The observed data points are corrected for absorption by the extragalactic background light assuming $\mathrm{z}=0.536$. The dashed lines represents the extrapolation fits of the Fermi data, using log-parabola (LP) and power-law (PL) functions. The shaded yellow area represents an extrapolation of the flux enhancing during the studied flares. Finally, the coloured solid lines indicates the neutrino upper-limits for different spectral indexes (from $E^{-3}$ in red to $E^{-1}$ in blue), with the ranges corresponding to the 5-95\% range of the energy sensitive to ANTARES.

\section{Conclusions}

In this contribution are shown the results of the extended time-dependent search for cosmic neutrinos from blazars using the data taken with the full 12 line ANTARES detector between 2008 and 2012. This search is supported on the time constriction from the gamma ray variable emission of the sources as seen by Fermi satellite and IACTs. Multiple neutrino spectra has been tested and a lag between the neutrino and gamma ray emission has been considered in the analysis. The most significant correlation was found with a GeV flare of the blazar 3C279 for which one neutrino event was detected in time/spatial coincidence with the gamma ray emission. However, this event had a post-trial probability of $67 \%$ and is thus background compatible. Upper-limits were obtained on the neutrino fluence for the selected sources and compared with the gamma ray observed fluxes. These comparisons show that for the brighter blazars, the neutrino flux limits are in the same order of magnitude as the high-energy gamma ray fluxes. Given this consideration, these searches are quite promising with further years of ANTARES data and for the future KM3NeT detector. A paper with these results is in the final stages of preparation. 


\section{References}

[1] J.K. Becker 2008, Phys. Rep., 458, 173.

[2] S.D. Bloom, A.P. Marscher, 1996, ApJ, 461, 657; L. Maraschi, G. Ghisellini, A. Celotti 1992, ApJL, 397, L5; C.D. Dermer, R. Schlickeiser, 1993, ApJ, 416, 458; M. Sikora, M.C. Begelman, M.J. Rees, 1994, ApJ, 421, 153.

[3] T.K. Gaisser, F. Halzen, T. Stanev, Phys. Rep. 258 (1995) 173.

[4] J.G. Learned, K. Mannheim, Ann. Rev. Nucl. Part. Sci. 50 (2000) 679.

[5] C.M. Urry, P. Padovani, 1995, PASP, 107, 803.

[6] F. Halzen, D. Hooper, Rep. Prog. Phys. 65 (2002) 1025.

[7] K. Mannheim, A\&A, 269, 67, 1993.

[8] M. Böttcher, Astrophys. Space Sci. 309 (2007) 95.

[9] K. Mannheim, P.L. Biermann, 1992, A\&A,253,L21.

[10] M. Böttcher, A. Reimer, K. Sweeney, A. Prakash, 2013, apJ, 768,54.

[11] M. Reynoso, G.E. Romero, M.C. Medina, A\&A 545, (2012).

[12] A. Mücke et al., Astropart. Phys. 18(6) (2003) 593.

[13] A. Atoyan, C. Dermer, New Astron. Rev. 48(5) (2004) 381.

[14] A. Neronov, M. Ribordy, 2009, Phys.Rev., D80, 083008.

[15] A. Mücke, R.J. Protheroe, Proc. 27th Int. Cosmic Ray conf, arXiv:0105543.

[16] A. Mücke, R.J. Protheroe, Astropart. Phys. 15 (2011) 121.

[17] M. Ageron et al., Nucl. Instrum. Meth. A 656 (2011) 11-38.

[18] S. Adrian-Martinez et al., The Astrophysical Journal 760:53(2012).

[19] J.A. Aguilar et al., Phys. Lett. B 696 (2011) 16-22.

[20] S. Adrian-Martinez et al., Proc. for the 33rd ICRC, Rio de Janeiro (2013), arXiv:1312.4308.

[21] A.A. Abdo et al.. 2010, ApJ, 722, 520.

[22] M. Ackermann et al., ApJ, 743 (2011) 171.

[23] T. Hovatta et al., MNRAS arxiv:1401.0538.

[24] S. Adrian-Martinez et al., Astropart. Phys. 36 (2012) 204-210.

[25] A. A. Abdo et al. 2013, ApJS, 208, 17. 
[26] A.A. Abdo et al. 2010, ApJ, 715, 429.

[27] S. Ciprini et al., 2011 Fermi Symposium proceedings - eConf C110509, arXiv:1111.6803. http://fermisky.blogspot.fr/

[28] http://fermi.gsfc.nasa.gov/cgi-bin/ssc/LAT/LATDataQuery.cgi.

[29] J.D. Scargle, The Astrophysical Journal Supplement Series, 45, 1-71, 1981; http://trotsky.arc.nasa.gov/ jeffrey/.

[30] J.D. Scargle, Astrophys. J., 504, 1998, 405-418.

[31] J.D. Scargle et al., Astrophys.J. 764 (2013) 167.

[32] J. Neyman, 1937, Phil. Trans. Royal Soc. London, Series A, 236, 333.

[33] M.G. Aartsen et al, submitted to ApJ, arXiv:1503.00598

[34] J. Aleksic et al, A\&A 530, A4 (2011).

[35] J. Aleksic et al, A\&A 567, A41 (2014).

[36] S.R. Kelner, F.A. Aharonian, V.V. Bugayov, 2006, Phys. Rev. D, 74, 034018.

[37] S.R. Kelner, F.A. Aharonian, 2008, Phys. Rev. D, 78, 034013.

[38] S. Buson, ATEL \#5680 (2013).

[39] S. Ciprini, J. Becerra Gonzalez, \#6036 (2014). 